\title{
Enhanced Cellular Uptake with a Cobaltacarborane- Porphyrin-HIV-1 Tat 48-60 Conjugate
}

Martha Sibrian-Vazquez, Erhong Hao, Timothy J. Jensen and M. Graça H. Vicente ${ }^{I}$

Department of Chemistry, Louisiana State University, Baton Rouge LA, 70803, USA.

E-mail : vicente@1su.edu

\section{Supporting Information}

HPLC Traces

$2-4$

Subcellular Localization Figures

$5-7$

${ }^{1}$ Corresponding author. Phone: (225) 578 7405, Fax: (225) 578 3458, E-mail : vicente@1su.edu 


\section{HPLC Traces}

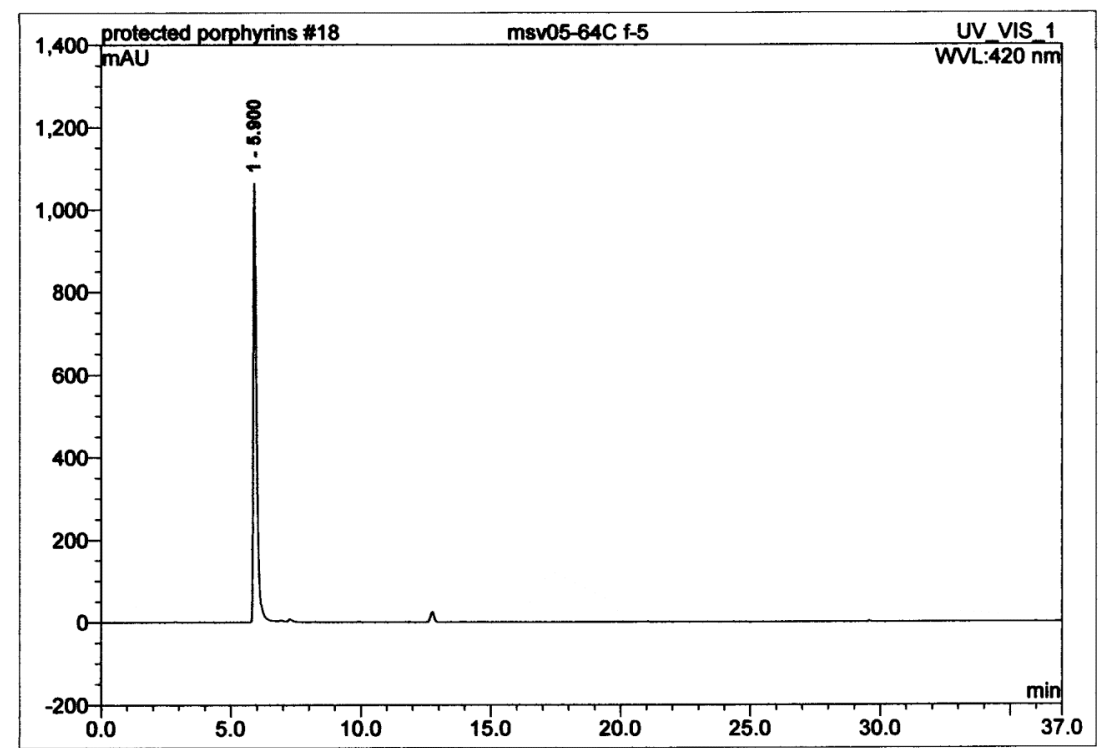

Figure 1. HPLC trace for porphyrin 3. Column: Delta Pak $C_{18} 300 \AA, 5 \mu \mathrm{m}, 3.9$ x 150 mm (Waters, USA); flow rate $1 \mathrm{~mL} / \mathrm{min}$; injected volume $20 \mu \mathrm{L}$; wavelength detection: $420 \mathrm{~nm}$. Stepwise gradient from $40 \%$ to $95 \%$ mobile phase B. Mobile phase A: $5 \%$ acetonitrile, $0.1 \%$ TFA, $\mathrm{H}_{2} \mathrm{O}$. Mobile phase B: $5 \% \mathrm{H}_{2} \mathrm{O}, 0.1 \%$ TFA, acetonitrile.

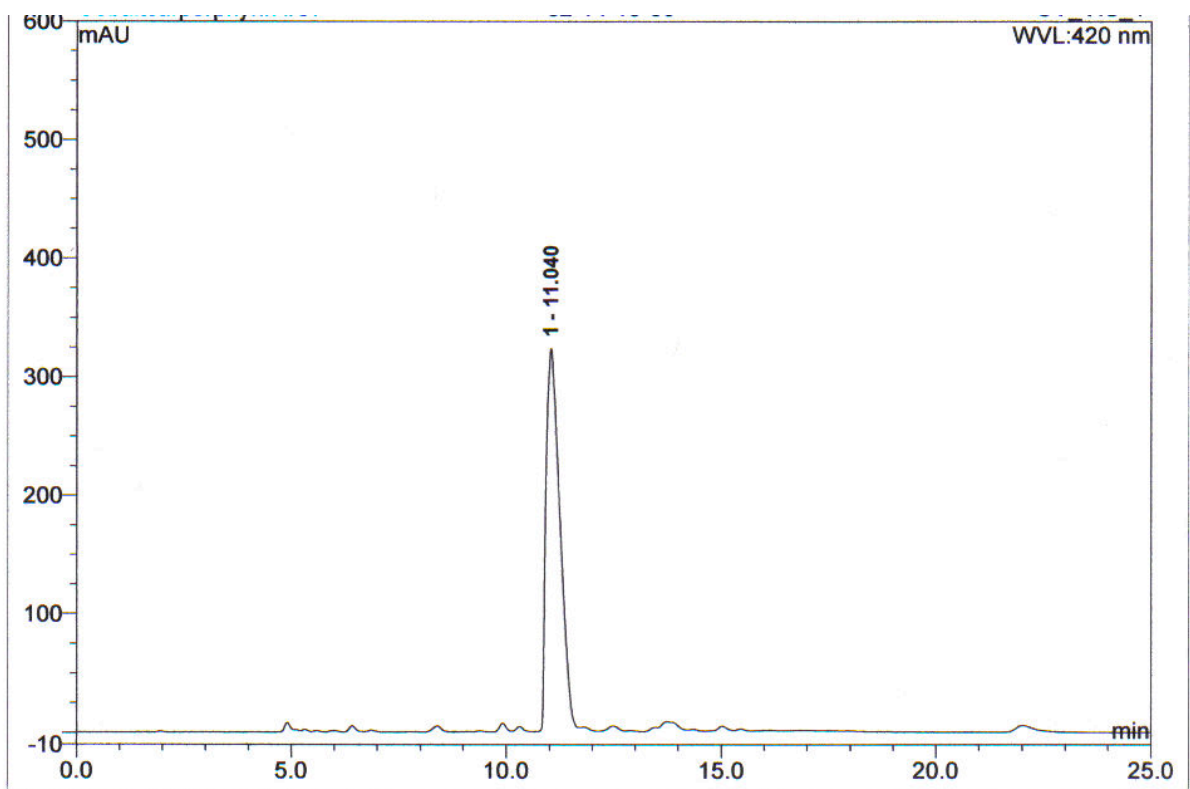

Figure 2. HPLC trace for conjugate 4. Column: Delta Pak $\mathrm{C}_{18} 300 \AA$, $5 \mu \mathrm{m}, 3.9$ x $150 \mathrm{~mm}$ (Waters, USA); flow rate $1 \mathrm{~mL} / \mathrm{min}$; injected volume $20 \mu \mathrm{L}$; wavelength detection: $420 \mathrm{~nm}$. Stepwise gradient from $85 \%$ to $95 \%$ mobile phase B. Mobile phase A: $5 \%$ acetonitrile, $0.1 \%$ TFA, $\mathrm{H}_{2} \mathrm{O}$. Mobile phase B: $5 \% \mathrm{H}_{2} \mathrm{O}, 0.1 \%$ TFA, acetonitrile. 


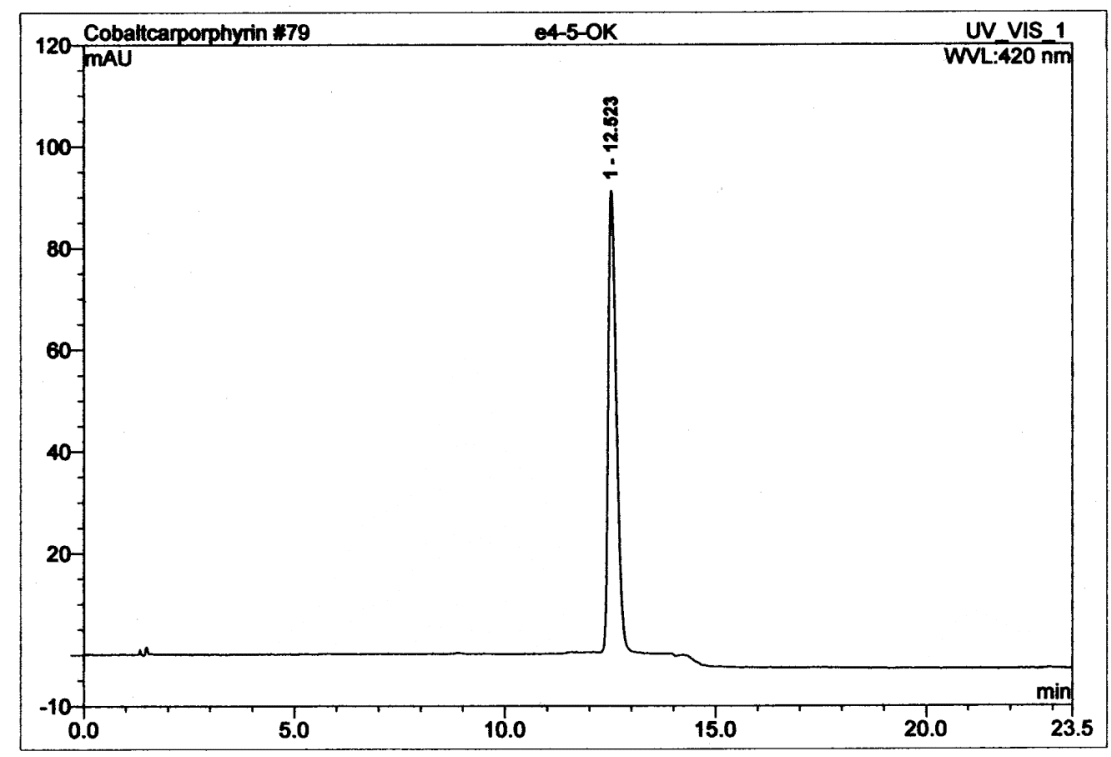

Figure 3. HPLC trace for Boc-protected conjugate 5. Column: Delta Pak $C_{18} 300 \AA, 5 \mu \mathrm{m}, 3.9 \times 150$ $\mathrm{mm}$ (Waters, USA); flow rate $1 \mathrm{~mL} / \mathrm{min}$; injected volume $20 \mu \mathrm{L}$; wavelength detection: $420 \mathrm{~nm}$. Stepwise gradient from $85 \%$ to $95 \%$ mobile phase B. Mobile phase A: $5 \%$ acetonitrile, $0.1 \%$ TFA, $\mathrm{H}_{2} \mathrm{O}$. Mobile phase B: $5 \% \mathrm{H}_{2} \mathrm{O}, 0.1 \%$ TFA, acetonitrile.

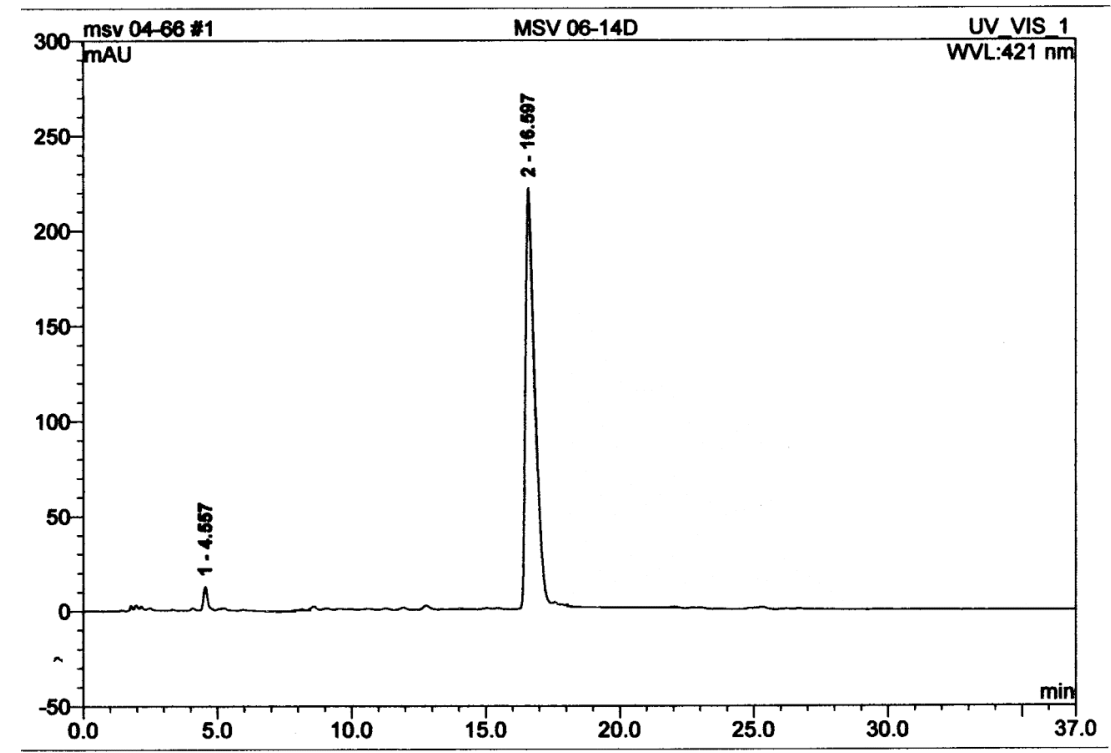

Figure 4. HPLC trace for conjugate 5. Column: Delta Pak $\mathrm{C}_{18} 300 \AA, 5 \mu \mathrm{m}, 3.9$ x $150 \mathrm{~mm}$ (Waters, USA); flow rate $1 \mathrm{~mL} / \mathrm{min}$; injected volume $20 \mu \mathrm{L}$; wavelength detection: $420 \mathrm{~nm}$. Stepwise gradient from $80 \%$ to $95 \%$ mobile phase B. Mobile phase A: $5 \%$ acetonitrile, $0.1 \%$ TFA, $\mathrm{H}_{2} \mathrm{O}$. Mobile phase B: $5 \% \mathrm{H}_{2} \mathrm{O}, 0.1 \%$ TFA, acetonitrile. 


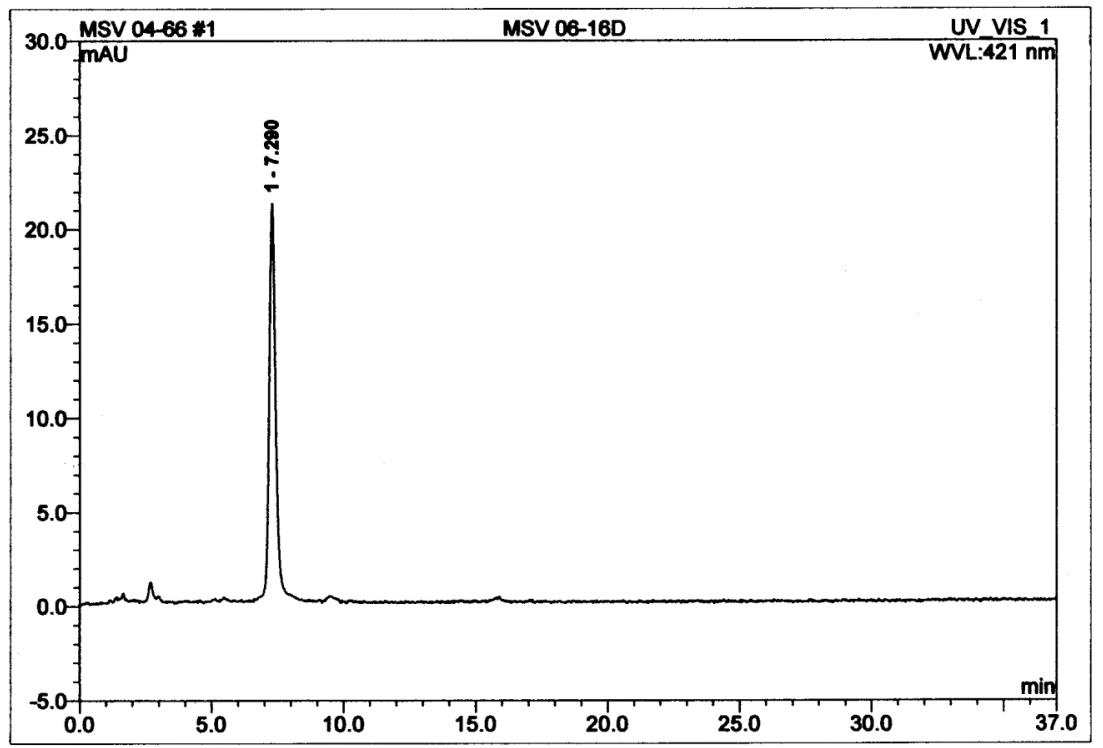

Figure 5. HPLC trace for conjugate 6. Column: Delta Pak $\mathrm{C}_{18} 300 \AA, 5 \mu \mathrm{m}, 3.9$ x $150 \mathrm{~mm}$ (Waters, USA); flow rate $1 \mathrm{~mL} / \mathrm{min}$; injected volume $20 \mu \mathrm{L}$; wavelength detection: $420 \mathrm{~nm}$. Stepwise gradient from $80 \%$ to $95 \%$ mobile phase B. Mobile phase A: $5 \%$ acetonitrile, $0.1 \%$ TFA, $\mathrm{H}_{2} \mathrm{O}$. Mobile phase B: $5 \% \mathrm{H}_{2} \mathrm{O}, 0.1 \%$ TFA, acetonitrile.

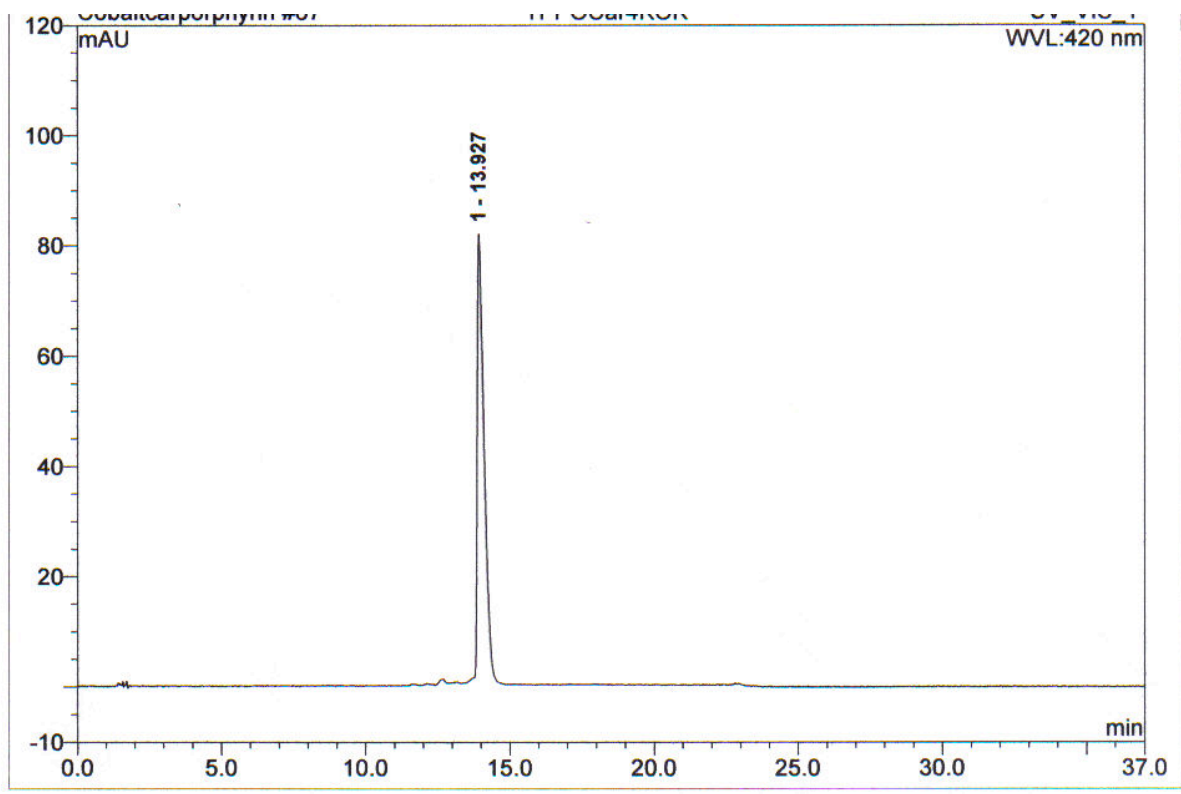

Figure 6. HPLC trace for conjugate 7. Column: Delta Pak $\mathrm{C}_{18} 300 \AA, 5 \mu \mathrm{m}, 3.9$ x $150 \mathrm{~mm}$ (Waters, USA); flow rate $1 \mathrm{~mL} / \mathrm{min}$; injected volume $20 \mu \mathrm{L}$; wavelength detection: $420 \mathrm{~nm}$. Stepwise gradient from $85 \%$ to $95 \%$ mobile phase B. Mobile phase A: $5 \%$ acetonitrile, $0.1 \%$ TFA, $\mathrm{H}_{2} \mathrm{O}$. Mobile phase B: $5 \% \mathrm{H}_{2} \mathrm{O}, 0.1 \%$ TFA, acetonitrile 


\section{Subcellular Localization}
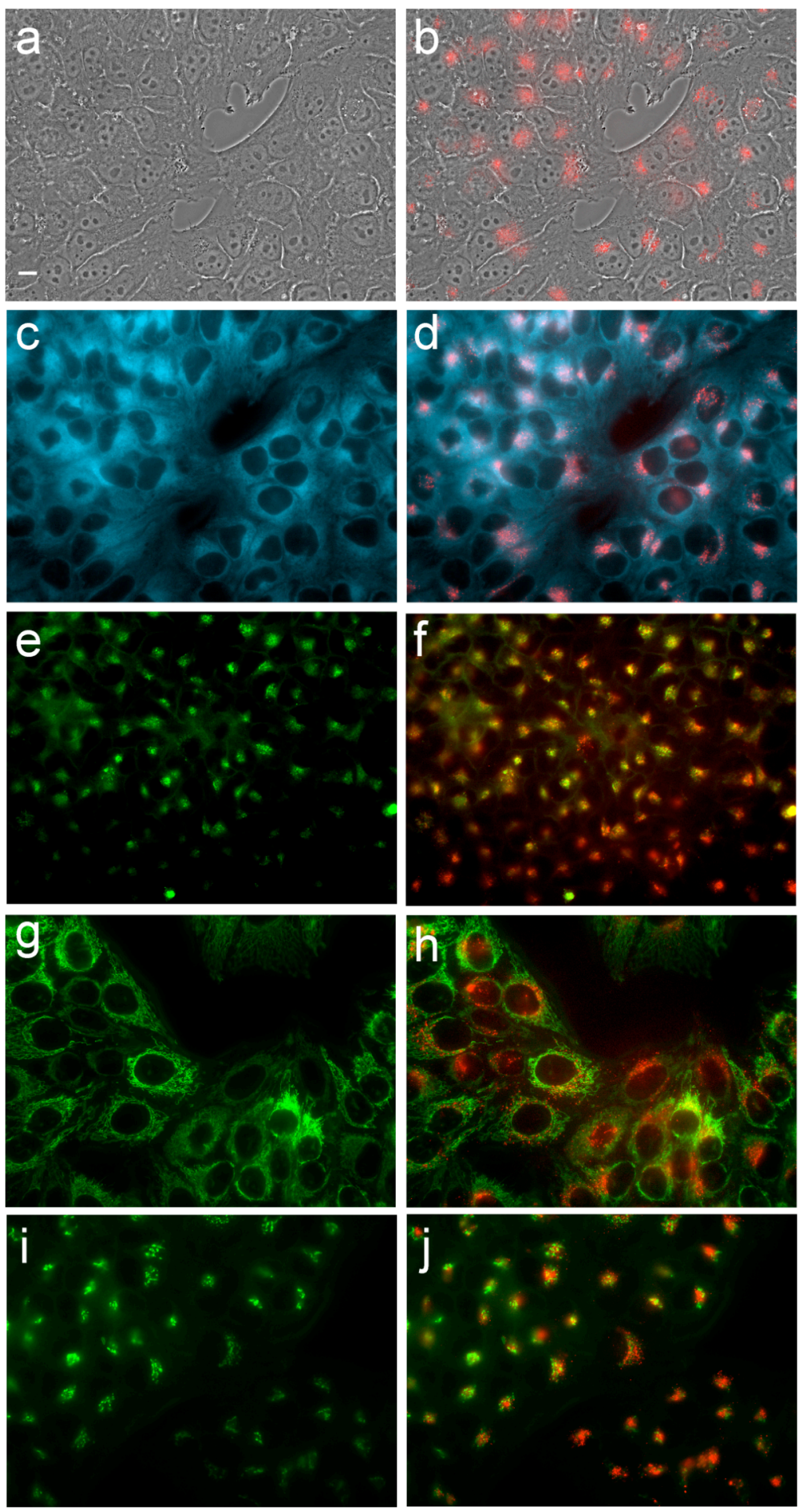

Figure 7. Subcellular localization of conjugate 4 in HEp2 cells at $10 \mu \mathrm{M}$ for $18 \mathrm{~h}$. (a) Phase contrast, (b) Overlay of 4 fluorescence and phase contrast, (c) ER Tracker Blue/White fluorescence, (e) LysoSensor Green fluorescence, (g) MitoTracker Green fluorescence, (i) BODIPY Ceramide, (d), (f), (h), and (j) overlays of organelle tracers with 4 fluorescence. Scale bar: $10 \mu \mathrm{m}$. 

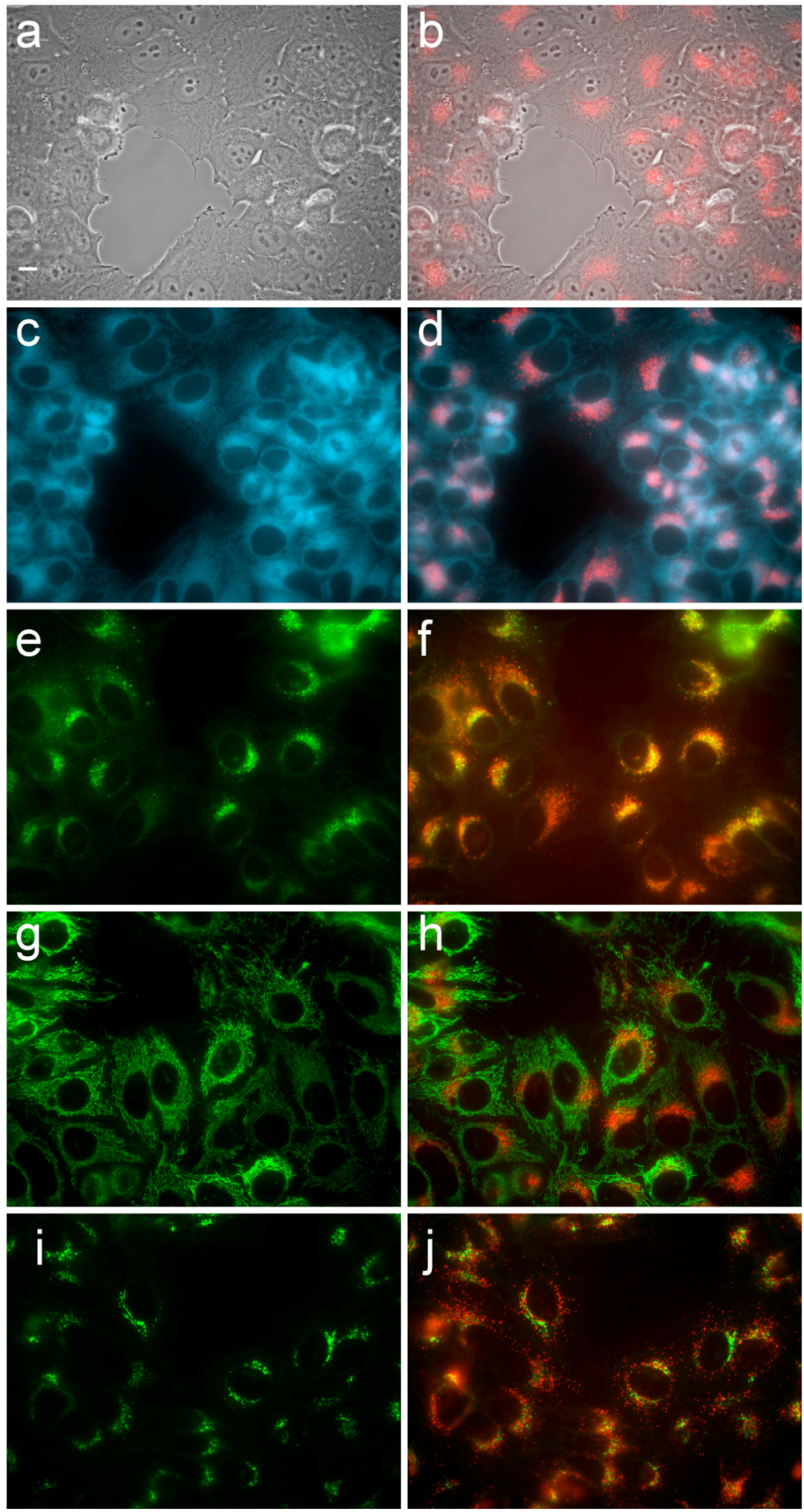

Figure 8. Subcellular localization of conjugate 5 in HEp2 cells at $10 \mu \mathrm{M}$ for $18 \mathrm{~h}$. (a) Phase contrast, (b) Overlay of 5 fluorescence and phase contrast, (c) ER Tracker Blue/White fluorescence, (e) LysoSensor Green fluorescence, (g) MitoTracker Green fluorescence, (i) BODIPY Ceramide, (d), (f), (h), and (j) overlays of organelle tracers with 5 fluorescence. Scale bar: $10 \mu \mathrm{m}$. 

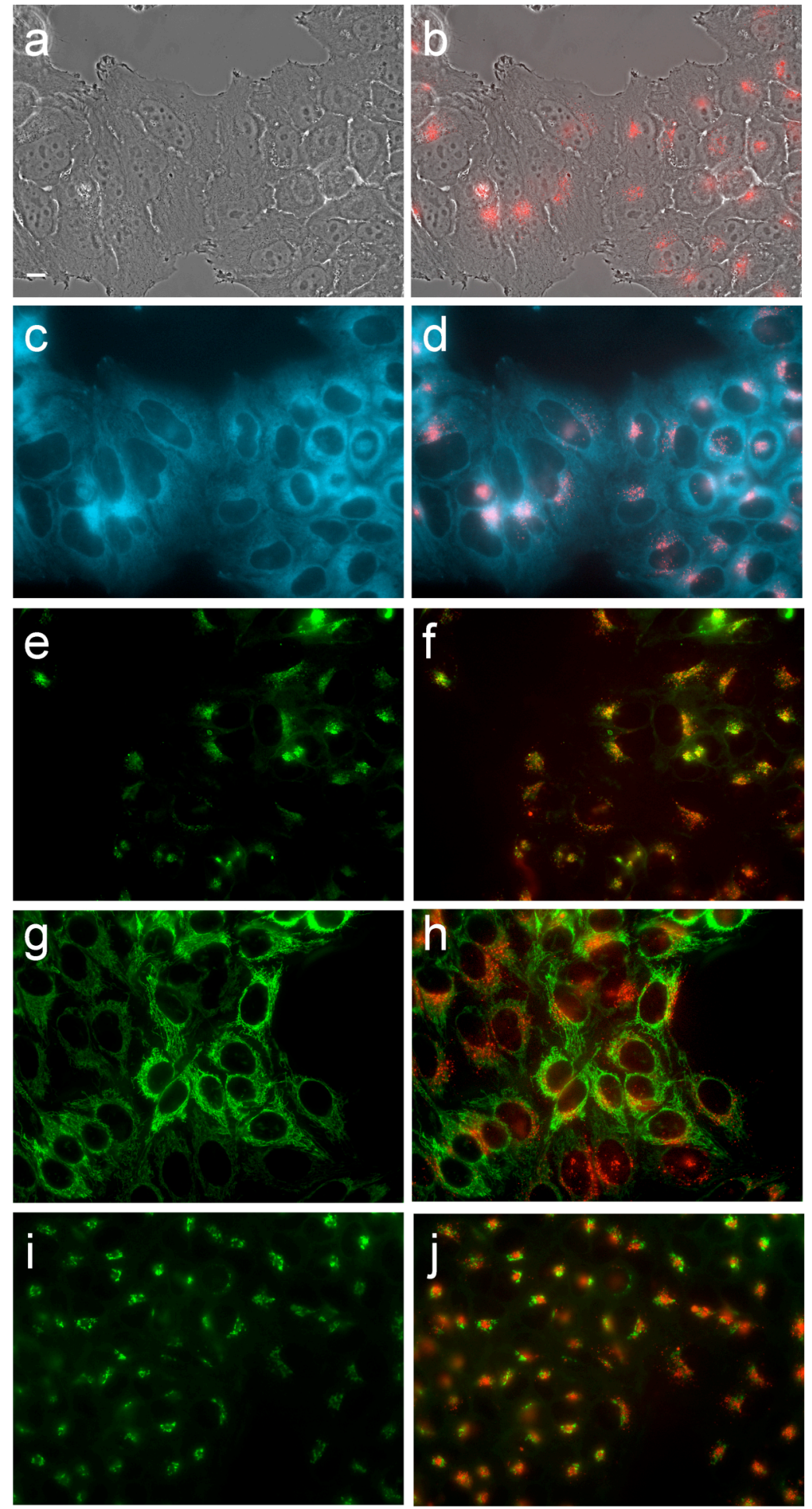

Figure 9. Subcellular localization of conjugate 7 in HEp2 cells at $10 \mu \mathrm{M}$ for $18 \mathrm{~h}$. (a) Phase contrast, (b) Overlay of 7 fluorescence and phase contrast, (c) ER Tracker Blue/White fluorescence, (e) LysoSensor Green fluorescence, (g) MitoTracker Green fluorescence, (i) BODIPY Ceramide, (d), (f), (h), and (j) overlays of organelle tracers with 7 fluorescence. Scale bar: $10 \mu \mathrm{m}$. 